\title{
Relocation puts elderly nursing home residents at risk of stress, although the stress is short lived
}

Hodgson N, Freedman VA, Granger DA, et al. Biobehavioral correlates of relocation in the frail elderly: salivary cortisol, affect, and cognitive function. J Am Geriatr Soc 2004;52:1856-62.

What are the effects of moving elderly people to a new nursing home?

\section{METHODS}

Design: Randomised controlled trial.

Setting: Long term nursing homes in greater Philadelphia and

Horsham, Pennsylvania between June 2001 and spring 2002.

of Population: 77 nursing home residents aged 65 and over.

Assessment: Participants were divided into two groups: residents moving to a new nursing home, and residents remaining in the Philadelphia nursing home. Baseline measurements were recorded in July and August 2001. Residents were moved during fall 2001, measurements were taken 1 week before moving, and 1 week and 4 weeks after moving.

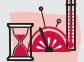

Outcomes: Mini-Mental State Examination scores, Observed Affect Rating Scale scores, salivary cortisol, blood pressure, and pulse.

Follow up period: Outcomes were assessed 1 week before the move, and 1 week and 4 weeks after the move.

\section{MAIN RESULTS}

1 week before moving: residents moving had a significantly greater increase from baseline in late afternoon cortisol compared with residents remaining $(\mathrm{p}=0.015)$ l week after moving: residents moving had a significantly greater increase from baseline in early morning cortisol and mild pleasure, although they had a greater reduction in pulse, anxiety, and sadness compared with residents remaining (early morning cortisol: $\mathrm{p}=0.005$; mild pleasure: $\mathrm{p}=0.042$; pulse: $\mathrm{p}=0.002$; anxiety: $\mathrm{p}=0.027$; sadness: $\mathrm{p}=0.003$ ). 4 weeks after moving: residents moving had a significantly greater reduction from baseline in late afternoon cortisol, pulse, anxiety, and sadness compared with residents remaining (late afternoon cortisol: $\mathrm{p}=0.030$; pulse: $\mathrm{p}=0.002$; anxiety: $\mathrm{p}=0.002$; sadness: $\mathrm{p}=0.005$ )

\section{CONCLUSIONS}

Elderly nursing home residents may experience stress before relocation, although the stress is short lived. Quality of care can be improved by preparing residents for relocation, by identifying and alleviating stressors associated with relocation.

For correspondence: Dr Nancy Hodgson, Senior Research Scientist, Polisher Research Institute, Madlyn and Leonard Abramson Center for Jewish Life, 1425 Horsham Road, North Wales, PA 19454, USA; nhodgson@abramsoncenter.org

Sources of funding: National Institute on Aging Grant R03 AG19896-01, the Pennsylvania State University College of Health and Human Development and Population Research Institute, and the Polisher Research Institute.
NOTES

Results should be treated with caution. The study conducted multiple analyses for different outcomes, which increases the likelihood that significant results would have arisen by chance.

\section{Commentary}

$\mathrm{H}$ dgson et al examined the effects of moving nursing home esidents from one environment to another. Staff in skilled nursing and other long term care facilities, as well as family members, commonly believe that frail older adults experience adverse physical, psychological, and cognitive effects from the stress of relocation. Whereas several early studies found adverse effects post-transfer, ${ }^{1}{ }^{2}$ more recent investigations examining a range of health outcomes have concluded that relocation produces no ill effects. Indeed, some argue that a move to an improved physical environment has positive effects on residents' wellbeing. ${ }^{3}$

Hodgson et al make an important contribution to the literature on the controversy regarding the direction, nature, and extent of relocation effects in nursing home residents. The first randomised controlled trial to examine this issue, the study involved a naturally occurring experiment where residents were relocated to a new facility over a 7 month period. A move preparation programme provided information and support to those who agreed to the relocation. The principal outcome measure was salivary cortisol, a sensitive, non-invasive assessment of a physiological marker of the stress response. ${ }^{4}$ Repeated measures were also made of cognitive status, observed affect, blood pressure, and pulse rate. Compared with the group who had not yet moved, relocated residents exhibited higher cortisol levels 1 week after the move followed by a significant decline at 4 weeks post-transfer. At this later point, those who had relocated also had lower pulse rates and significantly fewer symptoms of depression and anxiety than the controls. No cognitive response to the move was found.

Because the sample was homogeneous with respect to race and culture, the study's generalisability is limited. The signed consent rate was only slightly more than $50 \%$, and the study authors do not report comparisons of the clinical characteristics of the refusals and the participants. In addition, xerostomia (dry mouth), common in nursing home residents, prevented one-third of those enrolled in the trial from providing analysable saliva samples. Nonetheless, the findings suggest that with preparation beforehand and assistance in the early weeks posttransfer, stress can be minimised; if the move is to a more pleasant, supportive environment, residents' experience may be positive. Additional research is needed with other populations and to further explore the relationship between changes in affect and the stress response as measured by sensitive biomarkers.

Allyson M Washburn, PhD Saybrook Graduate School and Research Center, San Francisco CA USA

1 Markus $E$, Blenker $M$, Bloom $M$, et al. The impact of relocation upon mortality rates of institutionalized aged person. J Gerontol 1971;26:53741.

2 Coffman TL. Relocation and survival of institutionalized aged: a reexamination of the evidence. Gerontologist 1981;21:483-500.

3 Mirotznik J, Kamp LL. Cognitive status and relocation stress: a test of the vulnerability hypothesis. Gerontologist 2000:40:531-9.

4 Kirschbaum C, Hellmammer DH. Salivary cortisol. Encycl Stress 2000;3:379-84 